\title{
Neumonía necrotizante asociada a infección por influenza $A$ H1N1 en un niño con mucopolisacaridosis tipo II. Caso clínico
}

\author{
Necrotizing pneumonia associated with influenza A H1N1 infection in a child \\ with mucopolysaccharidosis type II. Case report
}

\author{
Dr. Daniel A. Huillca-Huerta ${ }^{a, b}$, Estud. Luis G. Yllescas-Estela, ${ }^{a,}$, Dra. Elizabeth A. Huerta-Medina ${ }^{a}$ y \\ Dr. Pedro E. San Martín-Howard ${ }^{a, b}$
}

\begin{abstract}
RESUMEN
La mucopolisacaridosis tipo II (MPS II) es un desorden recesivo ligado al cromosoma $\mathrm{X}$, caracterizado por una deficiencia de la enzima iduronato-2-sulfatasa, que lleva a una afectación multisistémica por acumulación tisular de los glicosaminoglicanos heparan y dermatan sulfato. Reportamos el caso de un niño de 9 años diagnosticado con MPS II a los 4 años de edad, catalogado como portador de una variante grave. Presentó neumonía necrotizante asociada a infección A H1N1, que requirió ventilación mecánica por 1 mes y 10 días. Este caso enfatiza la importancia de que los trabajadores de la salud estén alerta a las potenciales complicaciones en pacientes con MPS II, tales como la infección por influenza A H1N1, la que, a su vez, puede estar asociada con neumonía necrotizante. Palabras clave: mucopolisacaridosis tipo II, influenza A H1N1, neumonía necrotizante.
\end{abstract}

\begin{abstract}
Mucopolysaccharidosis type II (MPSII) is an X-linked recessive disorder characterized by a deficiency of the enzyme iduronate2-sulfatase leading to a multisystem involvement by tissue accumulation of glycosaminoglycans heparan and dermatan sulfate. We report a case of a 9-year-old boy diagnosed with mucopolysaccharidosis type II at 4 years of age, classified as severe variant. He presented necrotizing pneumonia associated with influenza A H1N1 infection, requiring mechanical ventilation for 1 month and 10 days. This case emphasizes the importance of healthcare workers to be aware of potentially lethal complications in patients with MPS II, such as influenza A H1N1 infection, which in turn may be associated with necrotizing pneumonia.
\end{abstract}

Key words: mucopolysaccharidosis type II, influenza A H1N1, necrotizing pneumonia.

http:/ /dx.doi.org/10.5546/aap.2015.e1

a. Facultad de Medicina San Fernando, Universidad Nacional Mayor de San Marcos.

b. Departamento de Pediatría, Hospital Nacional Dos de Mayo.

c. Asociación para el Desarrollo de la Investigación Estudiantil en Ciencias de la Salud (ADIECS). Universidad Nacional Mayor de San Marcos. Lima, Perú.

Correspondencia:

Dr. Daniel A. Huillca-Huerta: daniel.huillca@gmail.com

Conflicto de intereses: ninguno que declarar.

Recibido: 16-12-2013

Aceptado: 20-8-2014

\section{INTRODUCCIÓN}

La mucopolisacaridosis tipo II (MPS II), o síndrome de Hunter, es una enfermedad de depósito lisosomal heredada en un patrón recesivo ligado al cromosoma $X$. Afecta primariamente a los varones y su incidencia global ha sido estimada en 1 por cada 170000 varones nacidos vivos. La deficiencia de la enzima iduronato-2-sulfatasa que subyace en la MPS II genera afectación multiorgánica por acumulación tisular progresiva de los glicosaminoglicanos heparan y dermatan sulfato. ${ }^{1}$

La infección por el virus de la influenza A H1N1 ha sido muy estudiada desde la pandemia de 2009. Estudios genéticos sugieren asociaciones de susceptibilidad con la gravedad en la infección con este virus. Se sospecha de una relación entre el alelo rs12252 y la infección por H1N1; ${ }^{2}$ sin embargo, se requieren estudios adicionales con muestras más grandes. Además, se ha encontrado una asociación entre polimorfismos en TLR3, en los genes FCGR2A, C1QBP y el riesgo incrementado de neumonía grave en niños infectados con A H1N1. ${ }^{3}$

Reportamos un caso de neumonía necrotizante que complica la infección por influenza A H1N1 en un niño peruano con mucopolisacaridosis tipo II grave. El caso constituiría el primer reporte que muestra esta asociación clínica. De este modo, enfatizamos la importancia de tener en cuenta las complicaciones infecciosas potencialmente mortales en pacientes con MPS II y su consecuente impacto en la calidad y esperanza de vida de estos pacientes.

\section{CASO CLÍNICO}

Paciente varón de 12 años de edad, que es traído a la emergencia del hospital por presentar marcada dificultad respiratoria. El paciente tuvo síntomas gripales progresivamente graves durante siete días y permaneció afebril. Fue tratado con nebulización con albuterol de 
2,5 mg cada 4 horas y un curso de 3 días de amoxicilina de $50 \mathrm{mg} / \mathrm{kg} /$ día en un centro de salud comunitario. Durante las 24 horas antes de su admisión, el niño desarrolló agitación, disnea marcada e intensa tos productiva con expectoración blanquecina. La madre había presentado intensos síntomas gripales algunos días previos al inicio de la enfermedad actual del niño.

El paciente tiene como antecedente el diagnóstico de mucopolisacaridosis tipo II a la edad de 4 años luego de que su facies tosca, manos en garra y retardo mental en el contexto de un cuadro de obstrucción bronquial recurrente llevaran a la sospecha clínica. Después de encontrársele un elevado nivel de glicosaminoglicanos en orina, un ensayo de actividad enzimática para MPS II confirmó el diagnóstico. Desde entonces, el paciente nunca ha recibido terapia de remplazo enzimático.

Desde los 6 años, él presenta una significativa regresión cognitiva y actualmente no habla ni camina. A los 7 años, presentó sarampión sin complicaciones. Además, presenta problemas de deglución, que han ido empeorando desde los 8 años de edad; en la actualidad, es alimentado por vía de una sonda nasogástrica. El paciente ha presentado episodios periódicos de obstrucción bronquial cada 3-4 meses, que requirieron manejo ambulatorio $\mathrm{u}$ hospitalizaciones de corta estadía sin llegar a complicaciones. El paciente había completado el esquema nacional de vacunación; no obstante, no había recibido la vacuna contra la influenza.

Los antecedentes familiares son positivos para un primo con MPS II grave.

En la admisión, el examen físico lo evidenció malnutrido, letárgico y en mal estado general. El paciente se encontraba con marcada agitación; presentaba taquicardia (100 lat/min), taquipnea (60/min), tirajes intercostal y subcostal; estaba pálido, afebril $\left(37,5^{\circ} \mathrm{C}\right)$, con presión arterial de $125 / 66 \mathrm{mmHg}$. Su peso era $21 \mathrm{~kg}\left(<5^{\text {to }}\right.$ percentil); su talla era $132 \mathrm{~cm}\left(<25^{\text {to }}\right.$ percentil). Se observaron dismorfismo facial, macrocefalia y macroglosia. Presentaba acropaquias, dedos en garra y múltiples contracturas articulares, así como lesiones cutáneas descamativas diseminadas. No se evidenciaban lesiones cutáneas en forma de guijarros o de color marfil. Úlceras de decúbito se evidenciaban en la región maleolar y trocantérea izquierdas.

El examen respiratorio reveló sibilantes espiratorios en el hemitórax derecho, murmullo vesicular disminuido y crépitos en el hemitórax izquierdo, roncos difusos en ambos hemitórax. Al momento de la percusión, se evidenció matidez bibasal con predominancia izquierda. El examen cardiovascular fue normal. En el examen abdominal, se encontró hepatomegalia sin más alteraciones.

En el examen neurológico, el paciente lucía letárgico, incapaz de mover ninguna extremidad, pero respondía a los estímulos y presentaba pupilas reactivas. Los reflejos osteotendinosos estaban conservados sin respuesta plantar extensora. Ninguna otra anormalidad fue detectada.

Los hallazgos anormales en las pruebas de laboratorio incluyeron hemoglobina sérica de 9,6 mg/dL; leucocitos: $22400 / \mathrm{mm}^{3}$; eosinófilos $2 \%$ : $448 / \mathrm{mm}^{3}$ (VN: 20-400/ $\mathrm{mm}^{3}$ ); basófilos 0\%: 0/ $\mathrm{mm}^{3}$ (VN: 0-150/ $\mathrm{mm}^{3}$ ); abastonados 8\%: 1792/ $\mathrm{mm}^{3}$ (VN: 0-700/ $\mathrm{mm}^{3}$ ); segmentados 67\%: 15008/ $\mathrm{mm}^{3}$ (VN: 1800-7500/ $\mathrm{mm}^{3}$ ); linfocitos 21\%: 4704/ $\mathrm{mm}^{3}$ (VN: $1500-4000 / \mathrm{mm}^{3}$ ); monocitos $2 \%$ : $448 /$ $\mathrm{mm}^{3}$ (VN: 0-800/ $\mathrm{mm}^{3}$ ); albúmina sérica de 2,15 $\mathrm{mg} / \mathrm{dL}$ (VN: 3,7-5,6 mg/dL); creatinina sérica de 0,2 mg/dL (VN: 0,8-1,5 mg/dL); urea sérica de 6 $\mathrm{mg} / \mathrm{dl}$ (VN: 19-43 mg/dL); proteína $\mathrm{C}$ reactiva de 214,9 mg/L (VN: 1-3 mg/L).

Los hallazgos en la radiografía de tórax (Figura 1) y la tomografía computarizada (TC) (Figuras 2 y 3) llevaron al diagnóstico de neumonía necrotizante. El ensayo de reacción en cadena de polimerasa en tiempo real de una muestra de hisopado faríngeo resultó positivo para influenza A H1N1. Un cultivo de secreción bronquial obtenida por fibrobroncoscopia resultó positivo para Pseudomonas aeruginosa y fue negativo para Mycobacterium tuberculosis. Se obtuvo un hemocultivo, que fue negativo.

Adicionalmente, una TC de columna cervical mostró una marcada estrechez de las vías aéreas compatible con traqueobroncomalasia (Figura 4).

El paciente requirió ventilación mecánica por 1 mes y 10 días; recibió tratamiento con osetalmivir de $60 \mathrm{mg}$ por vía oral cada 12 horas por 7 días, ciprofloxacina de $300 \mathrm{mg}$ por vía endovenosa cada 12 horas, vancomicina de 500 mg por vía endovenosa cada 12 horas, que llevó a la resolución clínica de la neumonía. En la última evaluación realizada 4 semanas después, el paciente respiraba espontáneamente, pero su condición general permanecía estacionaria. Se ha considerado necesaria la indicación de gastrostomía debido al considerable reflujo gastroesofágico que presenta y al subsecuente 
riesgo de aspiración; el pronóstico del paciente es reservado.

\section{DISCUSIÓN}

La mucopolisacaridosis tipo II tiene un importante impacto en la calidad de vida, a un nivel mayor que con otras enfermedades pediátricas crónicas. ${ }^{4} \mathrm{El}$ considerable declive neurológico de los pacientes con la forma grave, quienes constituyen el $75 \%$ del total de casos reportados, ${ }^{5}$ lleva a retardo mental, desórdenes conductuales y regresión del desarrollo, como fue evidenciado en nuestro paciente.
El fenotipo usualmente emerge en los primeros años de edad, aunque, en aquellos con la variante atenuada, se estima que puede ocurrir pocos años más tarde. Durante la infancia temprana, los pacientes pueden presentar hepatoesplenomegalia, hernia inguinal y umbilical, y facies tosca; ${ }^{6}$ esta última es un signo fuertemente sugerente de enfermedades de depósito lisosomal.

La afectación respiratoria es también un hallazgo para considerar dado que el depósito de glicosaminoglicanos en la región orofaríngea y traqueobronquial lleva a una obstrucción grave

FiguRA 1. (A) Radiografía de tórax. Opacidades irregulares bilaterales en áreas perihiliares con predominancia izquierda y áreas de irregular hiperlucencia. (B) Tomografía computarizada de tórax en plano axial. Cavidades parenquimales múltiples en el hemitórax izquierdo (flecha)
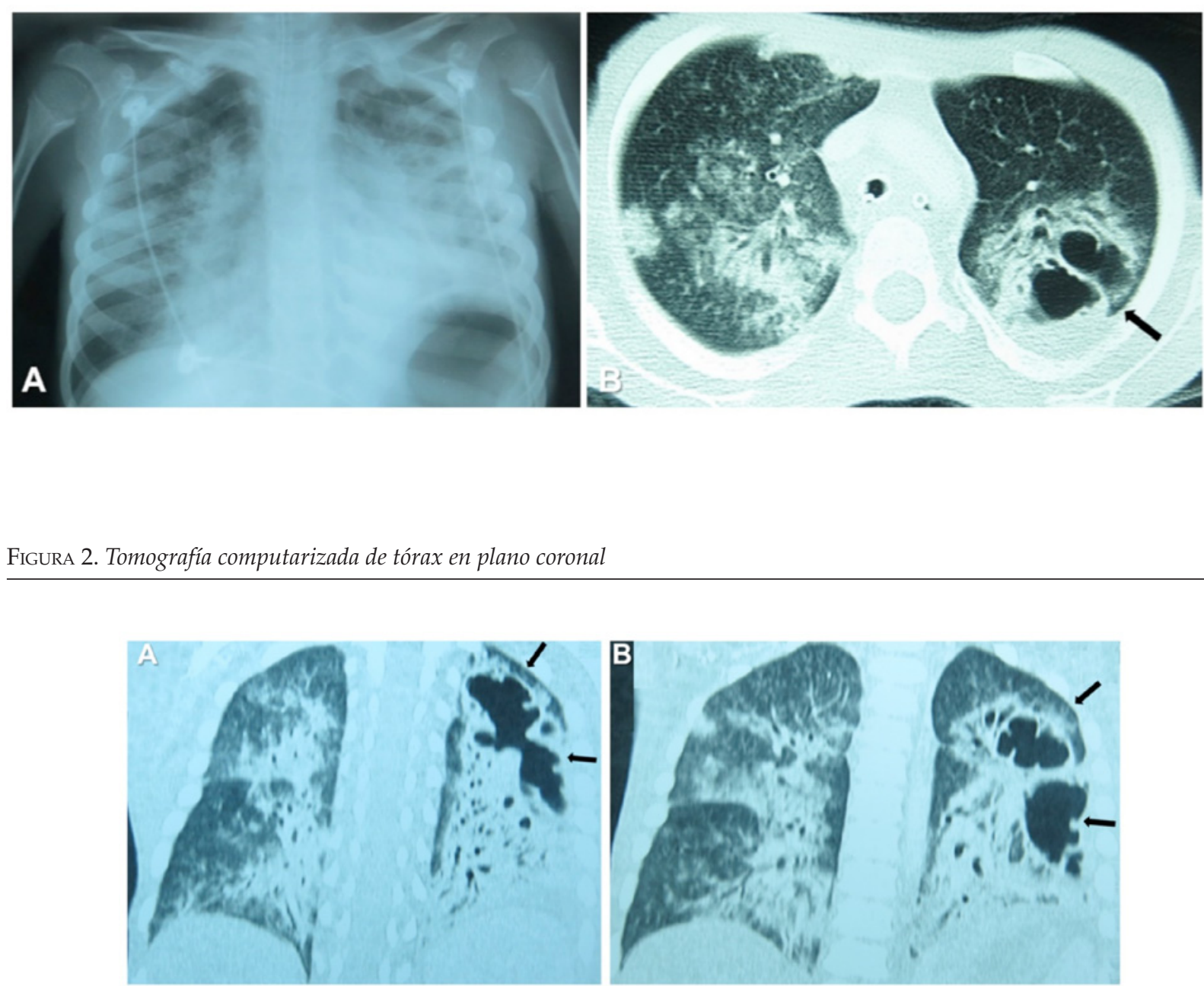

(A, B) Múltiples cavidades parenquimales de contornos irregulares y engrosados en el hemitórax izquierdo (flechas), consolidación bibasal con predominancia izquierda asociada a opacidades subpleurales y centroacinares en forma de cuña. 
de la vía aérea secundaria a macroglosia, estenosis supraglótica y traqueobroncomalasia, ${ }^{7}$ la que fue evidenciada en nuestro paciente. Posteriormente, puede llegar a generarse, incluso, una restricción pulmonar por depósito de glucosaminoglicanos en el esqueleto torácico.

En términos de expectativa de vida, los pacientes con MPS II con la variante grave infrecuentemente viven más allá de la segunda década. Complicaciones, como enfermedad pulmonar obstructiva, insuficiencia cardíaca, asfixia e infecciones, han sido identificadas como las principales causas de muerte. ${ }^{8}$

En el caso de nuestro paciente, la infección por influenza A H1N1 contribuyó significativamente a su actual deterioro, probablemente debido a alteraciones en el sistema respiratorio de los pacientes con mucopolisacaridosis, como una
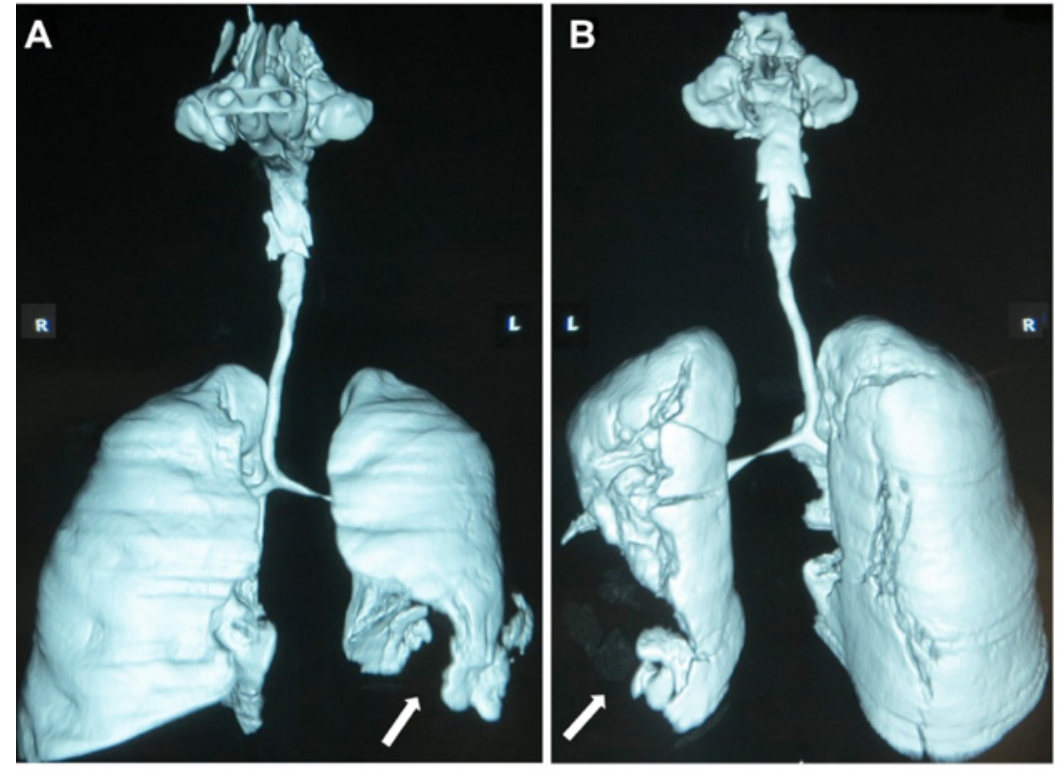

Cavidad parenquimal en pulmón izquierdo. (A) Vista anterior. (B) Vista posterior.

FIgURA 4. Tomografía computarizada de la columna cervical
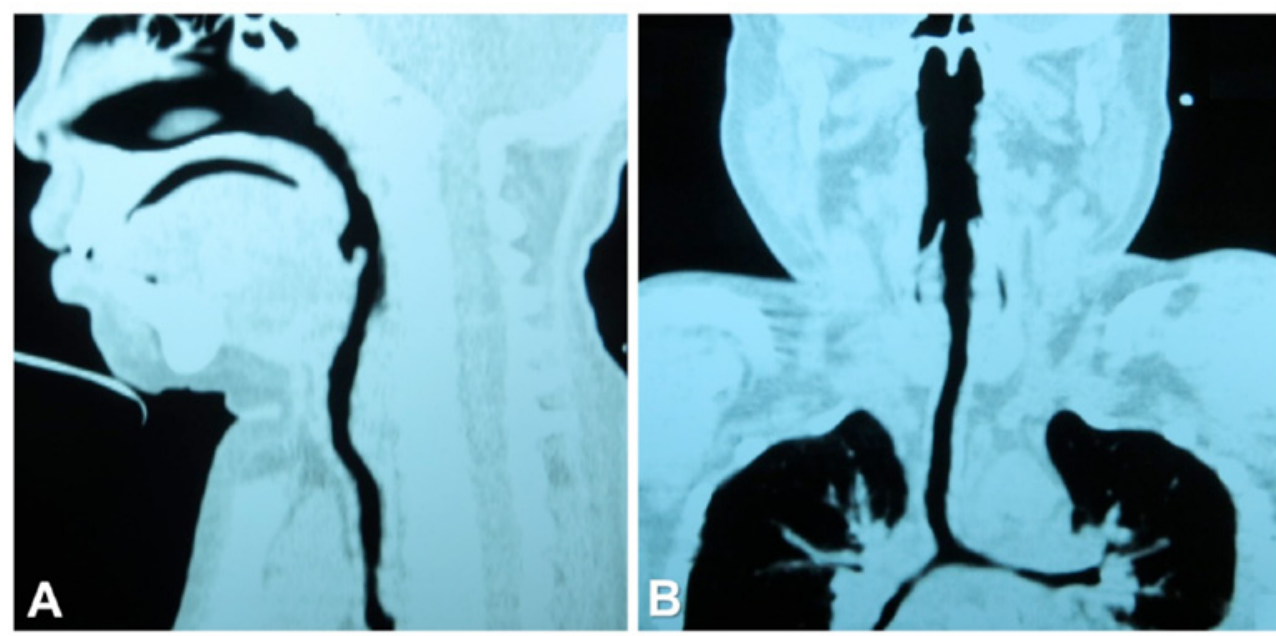

Marcado estrechamiento de las vías aéreas compatible con traqueobroncomalasia. (A) Vista sagital. (B) Vista coronal. 
capacidad ventilatoria disminuida, sumadas a las anormalidades encontradas en el tracto respiratorio, como distorsión de la tráquea o debilidad en los músculos espiratorios y un difícil aclaramiento de secreciones. ${ }^{9}$

Se ha descrito un mayor riesgo de adquirir la infección en personas con enfermedades cardíacas y con enfermedades respiratorias, como asma y neumonía bacteriana adquirida en la comunidad..$^{10}$ La influenza A H1N1 grave afecta con mayor frecuencia a los pacientes inmunocomprometidos. En la revisión de la literatura, encontramos que la neumonía necrotizante relacionada con infección A H1N1 en el contexto de la mucopolisacaridosis tipo II no ha sido reportada antes. Sin embargo, algunos casos de neumonía necrotizante en niños previamente sanos, quienes habían desarrollado infección por influenza A H1N1, han sido descritos y una superinfección con Streptococcus pneumoniae fue identificada con mayor frecuencia. ${ }^{11,12}$ Otros organismos relacionados con una neumonía necrotizante son el Stafilococcus aureus, Haemofilus influenza y Mycoplasma pneumoniae. ${ }^{13}$

Consideramos la posibilidad de que la neumonía necrotizante en nuestro paciente se haya producido como consecuencia del desarrollo de una coinfección con Pseudomonas aeruginosa; a pesar de no ser el organismo más frecuente, ha sido reportado anteriormente como causa de este tipo de neumonía. ${ }^{14}$

Nosotros, por lo tanto, destacamos la importancia de tomar en consideración y reconocer la coinfección bacteriana y la neumonía necrotizante en niños con MPS II con deterioro progresivo en el contexto de una infección por influenza A H1N1, con el fin de establecer un tratamiento médico adecuado.

Asimismo, hacemos énfasis en la recomendación de vacunar contra la influenza a los pacientes pediátricos con enfermedades de base $y$, en especial, a aquellos con malformaciones de las vías aéreas, como en el caso presentado, ${ }^{15}$ por las comorbilidades que esta infección conlleva en ellos.

\section{REFERENCIAS}

1. Martin R, BeckM, Eng C, Giugliani R, etal. Recognition and diagnosis of mucopolysaccharidosis II (Hunter syndrome). Pediatrics 2008;121(2):e377-86.

2. Mills TC, Rautanen A, Elliott KS, Parks T, et al. IFITM3 and susceptibility to respiratory viral infections in the community. J Infect Dis 2014;209(7):1028-31.

3. Zúñiga J, Buendía-Roldán I, Zhao Y, Jiménez L, et al. Genetic variants associated with severe pneumonia in A/ H1N1 influenza infection. Eur Respir J 2012;39(3):604-10.

4. Raluy-Callado M, Chen WH, Whiteman DA, Fang J, et al. The impact of Hunter syndrome (mucopolysaccharidosis type II) on health-related quality of life. Orphanet J Rare Dis 2013;8:101.

5. Young ID, Harper PS, Newcombe RG, Archer IM. A clinical and genetic study of Hunter's syndrome. 2. Differences between the mild and severe forms. J Med Genet 1982;19(6):408-11.

6. Beck M. Mucopolysaccharidosis Type II (Hunter Syndrome): clinical picture and treatment. Curr Pharm Biotechnol 2011;12(6):861-6.

7. Wraith JE, Scarpa M, Beck M, Bodamer OA, et al. Mucopolysaccharidosis type II (Hunter syndrome): a clinical review and recommendations for treatment in the era of enzyme replacement therapy. Eur J Pediatr 2008;167(3):267-77.

8. Jones SA, Almássy Z, Beck M, Burt K, et al. Mortality and cause of death in mucopolysaccharidosis type II-a historical review based on data from the Hunter Outcome Survey (HOS). J Inherit Metab Dis 2009;32(4):534-43.

9. Berger KI, Fagondes SC, Giugliani R, Hardy KA, et al. Respiratory and sleep disorders in mucopolysaccharidosis. J Inherit Metab Dis 2013;36(2):201-10.

10. Gill JR, Sheng ZM, Ely SF, Guinee DG, et al. Pulmonary pathologic findings of fatal 2009 pandemic influenza A/ H1N1 viral infections. Arch Pathol Lab Med 2010;134(2):23543.

11. Yazer J, Giacomantonio M, MacDonald N, Lopushinsky S. Severe necrotizing pneumonia in a child with pandemic (H1N1) influenza. CMAJ 2011;183(2):215-9.

12. Casalegno JS, Escuret V, Celard M, Bouscambert M, et al. Cas de pneumopathie nécrosante communautaire secondaire à la surinfection d'une grippe saisonnière H1N1 par un streptocoque invasif du groupe A. Med Mal Infect 2010;40(12):710-2.

13. Wong KS, Chiu CH, Yeow KM,Huang YC, et al. Necrotising pneumonitis in children. Eur J Pediatr 2000;159(9):684-8.

14. SawickiGS, LuFL, Valim C, Cleveland RH, etal. Necrotising pneumonia is an increasingly detected complication of pneumonia in children. Eur Respir J 2008;31(6):1285-91.

15. Patria MF, Tagliabue $C$, Longhi B, Esposito S. Influenza vaccination in children at high risk of respiratory disease. Ther Adv Vaccines 2013;1(1):21-31. 